somewhat, so that the patient was discharged with a pupil only moderately displaced and V. of $\frac{6}{6}$ partly with $+10 \mathrm{D}$. sph. $こ+3.5$ D. cyl. In the other case there was no excuse of imperfect action of eserin. Next day the pupil was found small, but elongated and somewhat displaced up. Eserin drops were continued only three times a day, for an insufficient period. As a result iris became visible at the surface of the globe a fortnight later, with a barely perceptible elevation at one point.

In conclusion, it is only fair to recall and to utilize one of the Sindhi's good points. He makes a more intelligent hospital patient than the average elsewhere in India. Thus he appreciates the best possible result, with the least possible risk, and to obtain this he does not object to a fairly prolonged after-treatment, including "needling" when necessary. Hence, in the new Karachi Eye Hospital, opened this year, the material is at hand wherewith to demonstrate to a sufficiently critical public the superiority of the more established methods over the intra-capsular extraction now so widely practised in Northern India. There are two essentials in any such attempt. (1) A fair number of supplementary needlings must be performed; and (2) the safety aftorded by maximal perchloride irrigation must be utilized always, both in the primary and in the secondary operation. Of great assistance also in this endeavour will be the extended practice of simple extraction, which seems possible if the promise shown above, by the judicious use of adrenalin, of cocain, and of eserin, is substantiated.

\title{
INJURY TO THE EYE FROM SPECTACLE GLASS
}

\author{
BY \\ D. V. Giri, D.O. (Oxon.), \\ EASTBOURNE.
}

Cases

CASE I.-On August 8, 1916, R- K- - aged 62 years, came to the hospital with the history that three days previously, while chopping wood, a piece flew up and hit his right glass, which broke and pierced the eye.

On examination, I found : R.E. ecchymosis just below the lower lid. Subconjunctival haemorrhage below the limbus corneae and between it and the internal canthus. Perforating wound of the cornea in the lower inner quadrant. Behind the perforation, where probably the iris is adherent, a small clot of blood in the anterior chamber, which is very shallow. Iris somewhat bulged forward. Pupil semi-dilated and inactive. $\mathrm{T}-$. The fundus, the details of which became visible only with the help of the strongly luminous 
electric ophthalmoscope, shows posterior staphyloma and other changes characteristic of high myopia. No sign of infection of the wound.

L.E. also myopic.

Ung. hydrarg. oxid. flav. c. atropin sulph. and boric fomentations three times a day and a padded shade to be tied on the eye, so as to keep it closed, were prescribed.

The following are my notes on his subsequent visits :-

August 9, 1916.-Pupil still semi-dilated. No sign of infection of the wound. The patient says he had a very good night and that "the eye feels a lot easier."

August 14, 1916.-The wound continues free from infection. Reaction no more than the repair of the wound would explain, Some signs of the wound getting vascularized. A faint streak of blood on the lens-the remains of the clot noted on the first visit. Pupil semi-dilated. Pupillary margin appears free.

August 18 and 22, 1916.-Further improvement noted.

August 29, 1916. - The surface of the wound has become infected. The eye cocainized and the wound cauterized with the electric cautery.

September 15, 1916.-Pupil well dilated. Ulcer healing slowly.

September 29, 1916.- Ulcer healed. The unevenness not yet repaired. Eye quiet.

CASE II.-This is a case which came under the observation of Mr. A. Zorab in 1913. According to his notes, on the evening of 30th December of that year, E- E-, aged 39, another high myope, came to the hospital complaining that a couple of hours previously, not having been able to see well in the dark, she had jammed her left eyeglass against the edge of a half-open door and hurt her eye. This was again a perforating injury with a linear opening at the limbus through which the iris had prolapsed. The patient was admitted into the hospital, the prolapsed iris excised, and the eye put on the same local treatment as in the previous case. The next day the anterior chamber was found re-formed and the cornea and aqueous were clear. On the third day a bead of vitreous was noticed to protrude from the wound and was excised. Because of her having had at intervals attacks of severe pain in the eye, suspicion of the likelihood of a piece of glass being present in the eye and pressing on the ciliary body occurred, and the eye was X-rayed. The skiagram was negative. The patient was later discharged from the hospital with the eye perfectly quiet. She has been seen since and the eye has remained quiet.

\section{Remarks}

The foregoing add to the list of similar cases published by Hans Lauber, Sydney Stephenson, Casey A. Wood, and others, referred 
to in the "Notes and Echoes" column of the Ophthalmoscobe for October, 1916.

Some of the interesting conclusions arrived at by F. B. Vreeland, who made a communication on the subject of injuries of the eye from broken spectacle glass to the Chicago Ophthalmological Society recently, derive support from the cases described above. That injuries from broken glasses are very rarely serious is probably because the force with which the broken piece impinges on the globe is hardly ever sufficient to permit of its injuring the lens, or travelling further and lodging in the vitreous. Perhaps perforation of the cornea, as in the instances under consideration, is as severe an injury as could occur, and the exemption of the wound from infection, as noted in the first patient, although he came under treatment three days after the accident, seems to point to the probability that owing to its smooth and polished surface and the general absence of dirt on it because of the necessity of cleaning it and keeping it clean, the spectacle lens does not harbour microbes, at any rate of the pathogenic kind, and is hence non-infective. In speaking of surgical instruments, Haab says that it has been proved that a well-polished instrument may be rendered germ-free or nearly so by simple mechanical rubbing ("Atlas and Epitome of Operative Ophthalmology," by O. Haab, of Zurich-authorized translation from the German, edited by G. E. de Schweinitz, of Philadelphia. P. 77, 1910). I need hardly point out that an optical lens such as is used for spectacles is as highly polished and smooth an instrument as one could think of.

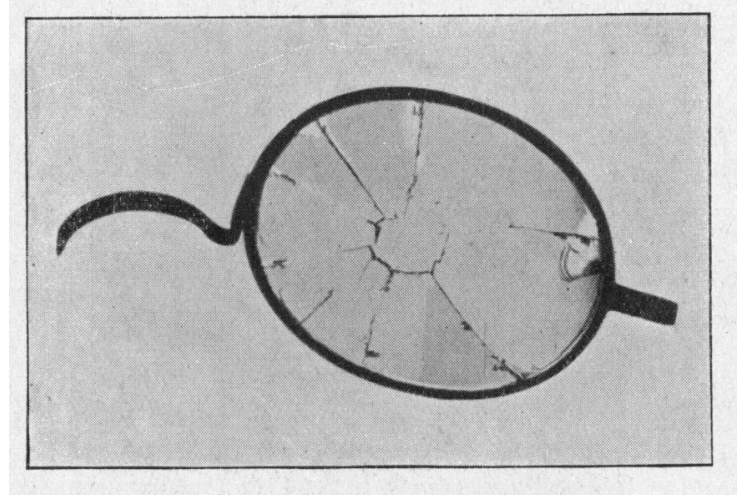

That minus lenses are more dangerous than plus ones appears to me to be due in the main to mechanical reasons. The concave lens is thinnest at the centre and gradually thickens centrifugally until it attains its maximum thickness at the periphery. The converse holds good of the convex lens. An impact on the rim of a concave lens strong enough to break it at its thickest part would obvióusly 
not leave the thin centre intact-because glass is highly elastic and brittle-and the piece that is smashed in, being thinnest at the centre, presents the sharp, more or less pointed end of the wedge it forms to the globe, and if the impetus is sufficient, easily perforates it. A much weaker impact on the central area would be sufficient to smash this part of the lens and drive small, thin, sharp fragments towards the globe. These mechanical considerations apply with special force to the higher minus lenses. The coming of the impact from in front, the resistance of the metal rim in the case of rimmed spectacles or of the metal catch in the case of the rimless ones to the backward motion of the thick peripheral edge, appear to determine the impinging of the thin sharp end of the wedge on the globe. The plus lens, because of its convex surface, does not in the first instance receive an impact very well. The striking body gets deflected in its course and dissipated in its force, just like a bullet striking a steel helmet. The blow is most likely to fall on its most prominent central part which is the thickest. Especially in the case of the higher plus lenses, unless the blow is strong enough independently to cause severe injury to the face or head, it is not likely to do anything more than at most crack the lens. In the case of a blow of such severity, the resulting damage of the face or eye would probably not be worse on account of the intervention of the lens. Even in a hypothetical case where, owing to an unapparent flaw in the glass, the lens gets smashed in by a comparatively weak blow, owing to the thickness of the impinging piece, it is unlikely that there would be anything more serious than a contusion or abrasion of some part of the eye. This contrast between the two kinds of lenses gets less and less as one goes down from the higher to the lower ones, although the balance always remains in favour of the plus lens.

Further, that these accidents are not confined to industrial occupations, but occur quite often in the course of athletic or domestic pursuits is evidenced by the fact that in Case I the eye was injured from broken spectacle glass because of a piece of wood hittıng it while the patient was chopping firewood, and in Case II, because of the patient jamming her left spectacle glass against the edge of a half-open door in the dark.

My thanks are due to Mr. Arthur Zorab, Surgeon to the Southampton Free Eye Hospital, for permission to publish the cases.

Since sending the above communication for publication I have had two cases which lend support to my interpretation of spectacle injuries.

The spectacles figured belong to a messenger boy.* While he was running an errand, he happened to pass through a street where

*The spectacles were exhibited at the Oxford Ophthalmological Congress of last year. 
there were some boys throwing stones at one another in play. One of the stones hit the left lens punching a hole in the middle and producing this interesting radiate fracture. It is a $-7 \mathrm{D}$. sph. It will be noticed that at its centre it is hardly $0.5 \mathrm{~mm}$. in thickness.

The impact could not have been very strong, as is evidenced by the retention of the fractured fragments in position in the frame and by the absence of any damage to the eye of the boy.

The next case is one of a man who, while watching a boat under repair at the Southampton Docks, had a bolt fly from the boat and strike his spectacles. The left lens was shivered to fine bits. He came in a hurry to the hospital fearing that something serious had happened to his left eye. All the injury he had sustained was a slight peripheral abrasion of the cornea. At the outer canthus and among the lashes there were a few fine flakes of glass which could easily pass for bits of broken cover slips such as are used in bacteriological microscopy.

The patient was a myope wearing $-4 \mathrm{D}$. spheres.

\title{
A NEW HETEROPHORIA TEST
}

\author{
BY \\ A. R. Brailey, \\ LONDON.
}

THIS simple apparatus was devised for the detection and estimation of heterophoria in candidates for the Royal Air Force. In use it has proved so rapid and efficacious that I think it may well supplant the Maddox rod in ordinary practice. In principle it depends on the old red and green letter test for binocular vision. Illuminated green letters are seen by one eye through a green glass, a red spot is seen by the other eye through a red glass. The letters are disposed in two rows, one above the other, and are placed so that the vertical limb of each letter in either row is at a distance, the one from the other, of the tangent of two degrees at six metres. Circular spots on the vertical limbs of each letter are at a distance of the tangent of half a degree from each other; on the horizontal limbs the distance of the tangent is one degree. The red spot, by a simple shutter, can be shifted to either of two positions. The position of the red spot as described by the patient in relation to the letters, at once denotes the presence, variety, and degree of heterophoria.

The advantages over the Maddox rod are:

1. That the amount of heterophoria is determined at once.

2. That by means of a rotating prism it is easy in a moment to see what correction is necessary to restore perfect muscle balance. 